\title{
Qu'est-ce qu'on voit quand on dit à ce que je vois ? À propos de l'évidentialité inférentielle et perceptuelle
}

\author{
Melissa Schuring $^{1 *}$ et Patrick Dendale ${ }^{1}$ \\ ${ }^{1}$ GaP (Grammar and Pragmatics) - Université d'Anvers - Belgique
}

\begin{abstract}
« Dans Une étude en rouge, lors de sa toute première rencontre avec Watson, Holmes lui annonce de but en blanc: "Vous êtes allée en Afghanistan, à ce que je vois !» L'information est bien évidemment correcte. Elle découle d'une série d'observations sur la personne de Watson, ainsi que d'inférences fondées sur des connaissances générales, dont l'association conduit à une unique explication, ce que Holmes appelle la déduction et qu'il fournit à Watson et aux lecteurs sans rien leur dire des étapes qui lui ont permis d'y aboutir. »"
\end{abstract}

\begin{abstract}
Résumé. Dans cet article, nous montrons que la suite de mots à ce que je vois peut être considérée comme un marqueur évidentiel, quand elle a le statut d'une collocation à fonction d'adverbial de phrase. Selon le contexte, elle marquera la perception directe (cas le moins fréquent) ou l'inférence sur la base d'indices perçus (cas de loin le plus fréquent). La collocation autorise aussi une interprétation de "perception limitée », signifiant "pour autant que je puisse voir ». Celle-ci s'explique par la présence implicite à l'intérieur de la collocation, ou explicite, dans sa variante à ce que je peux voir, du verbe modal pouvoir, à signification de «capacité ». La possibilité d'interprétation comme perception limitée amène pour l'emploi évidentiel inférentiel de la collocation, le caractère "défectible » (defeasible) de la conclusion inférée, qui, lui, est de nature à avoir une influence négative sur la fiabilité de l'information.
\end{abstract}

\begin{abstract}
What do we see when we say à ce que je vois? About inferential and perceptual evidentiality. In this article, we show that the sequence of words $\grave{a}$ ce que je vois can be considered a marker of evidentiality, when it has the status of a collocation with sentence adverbial scope. Depending on the context, à ce que je vois will mark direct perception (less frequent case) or inference based on perceived clues (most frequent case). The collocation also allows an interpretation of "limited perception", (meaning "as far as I can see"). This can be explained by the implicit presence within the collocation, or by the explicit presence in its variant à ce que je peux voir, of the modal verb pouvoir, meaning "capability". The interpretation of limited perception is responsible, with the inferential evidential use of the collocation, for the defeasible character of the conclusion, which is likely to have a negative influence on the reliability of the information.
\end{abstract}




\section{Introduction}

Dans cet article, nous présentons une analyse sémantique du groupe prépositionnel à ce que je vois. Dans le TLFi, cette suite de mots apparaît comme une sous-entrée de voir et y est qualifiée de locution. Nous nous interrogeons sur ce statut catégoriel. Que l'expression ait attiré notre attention, vient du fait qu'on peut y voir un marqueur évidentiel, proche de visiblement, qui, lui aussi, a été décrit comme marqueur évidentiel, notamment d'inférence (voir Dendale, Vanderheyden \& Izquierdo 2020 ; Vanderheyden \& Dendale 2018).

Notre étude se base sur un corpus de 500 exemples attestés de la suite de mots $\grave{a}+c e+q u e+j e+v o i s$, sélectionnés aléatoirement à partir des 8500 occurrences de cette suite que contient la base textuelle frTenTen12 de SketchEngine, complétés, pour les besoins de la démonstration, d'exemples de Frantext ou d'exemples cherchés ailleurs.

Dans la section $\S 1$, nous commençons par situer la suite de mots $\grave{a}+c e+q u e+j e+v o i s$ par rapport à toute une série de suites de mots quelque peu semblables, pour écarter d'abord celles qui ne feront pas l'objet de cette étude. Nous examinons ensuite (section 2) si la suite étudiée constitue bien une locution. Dans la section 3, nous étudions le caractère évidentiel de à ce que je vois. Dans la section 4, une comparaison de à ce que je vois avec sa variante à ce que je peux voir permet d'introduire l'idée de «limitation de la perception », dont nous examinons, dans la section 5, les conséquences pour l'emploi évidentiel inférentiel et, audelà, pour la fiabilité de l'information.

\section{Pour situer l'expression}

\subsection{Schéma de construction de l'expression sous étude}

L'expression à ce que je vois est composée des éléments suivants :

$$
\text { [Prép] ce que [PronPers] [Verbe] ([CCirc]) }
$$

Les quatre éléments entre crochets sont soumis à des variations.

La préposition initiale [Prép] à a comme variantes d'après (2) et selon (3) :

(2) Ok... on va dire que j'ai compris... mais en attendant, on fait quoi ? Car d'après ce que je vois, il n'est que dix heures du matin. (frTenTen12)

(3) Il y a un système de chat disponible, mais il est public. Pas moyen, selon ce que je vois, de créer un système de chat privé, si ton idée est d'utiliser le chat au lieu des MP. (frTenTen12)

Pour ce qui est du pronom personnel [PronPers], une recherche dans frTenTen12 a montré qu'il a comme variantes dans ce schéma $t u$, nous et $o n^{2}$, si les autres éléments restent les mêmes :

(4) A ce que tu vois, mes compagnons et moi sommes les mêmes... (frTenTen12)

(5) De fait, il arrive que la lutte défensive contre la motion pulsionnelle désagréable s'achève par la formation de symptôme ; à ce que nous voyons, c'est la possibilité dominante dans la conversion hystérique, mais en règle générale les choses suivent un autre cours (frTenTen12)

(6) Pour eux, il ne peut s'agir que d'une certitude soit physique, soit scientifique, soit historique, puisque, à ce qu'on voit, ils ne tolèrent pas même le doute. (frTenTen12)

C'est au niveau du verbe [Verbe] qu'il y a la plus grande variation. On trouve, tout d'abord, d'autres types de verbes de perception, comme lire (7), entendre / ouï dire (8) ou constater; mais aussi des verbes d'opinion ou de savoir, comme penser (9), croire, 
supposer, présumer, imaginer, comprendre, savoir ou se souvenir; des verbes d'apparence comme il semble ou il paraît (10), ou des verbes de communication comme dire (11), prétendre ou recevoir en guise de réponse ${ }^{3}$. Pour ce qui est du temps du verbe voir, le passé composé (12) et l'imparfait (13) sont attestés, à côté du présent :

(7) Ton avis, à ce que je lis, c'est que nous nous appauvrissions « un peu » pour que les autres s'enrichissent. (frTenTen12)

(8) Tu sais quoi ? Mon frère est mort. » «Sérieux ? Tu plaisantes ?! » « Non, il s'est fait tuer. » «Et comment ?» « ̀̀ ce que j'ai entendu dire, ce sont des chiens qui l'ont tué. » (frTenTen12)

(9) Le devoir du gouvernement, à ce que je pense, est d'exécuter la ligne qui doit joindre la mer à la frontière de Prusse. (frTenTen12)

(10) La maison nous a l'air petite ! A ce qu'il paraît cette sensation est normale à ce stade de la construction. (frTenTen12)

(11) Surtout, ne cherche pas à revoir Nathalie à ton retour. A ce qu'on m'a dit, elle est partie en voyage au Maroc avec un médecin. (frTenTen12)

(12) Tu n'as pas peur qu'il nous mange ? À ce que j'ai vu il a un appétit d'ogre... (frTenTen12)

(13) Je repris directement ma lecture. J'entendis des pas non loin de moi. Une jeune fille sautait de case en case. Des cases noires à ce que je voyais. (frTenTen12)

Pour finir, on trouve parfois un complément circonstanciel adjoint à la séquence ${ }^{4}$ :

(14) À ce que je vois sur le site, le positionnement de tes blocs est très mal fichu, et repose sur des marges négatives pour faire remonter les différents blocs. (frTenTen12)

\subsection{Constructions à exclure}

Le schéma de construction donné ci-dessus ne permet pas de délimiter suffisamment notre objet d'étude. Nous devons pour cela écarter deux cas de figure où la combinaison $\grave{a}+c e+q u e+j e+v o i s$ ne forme pas un tout, mais est composée de deux parties.

Le premier de ces cas est celui où l'on a d'un côté la préposition à et de l'autre le groupe pronominal ce que je vois; ce dernier peut remplir de nombreuses fonctions dans une phrase. Ne nous intéressent ici que celles où le groupe se combine avec la préposition $\grave{a}$ : complément déterminatif en à d'un adjectif (conforme à), complément indirect d'un verbe ou d'une locution verbale qui prend la préposition $\grave{a}$ (correspondre à, faire attention à) ou complément d'une locution prépositionnelle en $\grave{a}$ (par rapport $\grave{a}$, contrairement $\grave{a}$ ). Dans tous les cas la préposition à est commandée par un élément à sa gauche :

(15) Ce qu'il dit est conforme à ce que je vois et parfaitement cohérent avec la déduction la plus élémentaire qu'on peut en tirer. (frTenTen 12)

(16) Le seul mot qui pourrait correspondre à ce que je vois est: Magnifique. (frTenTen12)

(17) Je suis musicienne aussi mais je fais plus attention à ce que je vois qu'à ce que j'entends. (frTenTen12)

(18) J'exprime mon ressenti par rapport à ce que je vois à la télé en zappant comme un malade, et par rapport aux maigres informations en français accessibles sur certains sites. (frTenTen12)

(19) Comme à Durban, c'est frappant, contrairement à ce que je vois en France, ici les bateaux sont en mer en permanence à tirer des bords ou bien à régater. (frTenTen12) 
Le deuxième cas est celui où que n'est pas un pronom relatif, mais une conjonction, qui, en combinaison avec à ce ou jusqu'à ce, forme une conjonction complexe. Celle-ci lie je vois à un verbe, comme complément indirect (20) ou comme complément circonstanciel (21):

(20) On pourrait s'attendre à ce que je vois [sic] la question à travers des lunettes roses. (frTenTen12)

(21) Mais, jusqu'à ce que je vois [sic] cette assemblée aujourd'hui, je n'avais aucune idée du nombre de gens qui avait [sic] perdu un ami cher. (frTenTen12)

Dans ces deux cas, à ce que je vois ne constitue pas une unité soudée qui apparaît dans la phrase de façon autonome, comme dans tous les exemples précédents, mais une combinaison d'unités qui se rattache à un terme à gauche. Les combinaisons de ces types seront exclues de notre étude. Nous ne nous pencherons que sur l'unité que le TLFi considère comme une locution, nous demandant dans la section qui suit si ce terme convient.

\section{2. À ce que je vois : locution ou collocation?}

Dans les emplois sous étude ici, à ce que je vois est un groupe prépositionnel à fonction adverbiale, incident à une proposition ${ }^{5}$ (il est donc exophrastique dans la terminologie de Guimier $1996: 6$ ), proposition qui peut se trouver avant, après ou à l'intérieur de celle sur laquelle il porte 6 . Il est normalement séparé de cette proposition par une virgule et respecte, en tout cas en position initiale et interne, l'intonation typique du groupe rythmique (accentuation sur la dernière syllabe, les autres syllabes étant inaccentuées (Delattre 1939: 141)). Ainsi dans (22), à ce que je vois porte sur toute la proposition Je ne suis pas le seul à me retrouver ici en pleine nuit. La locution permet ici au locuteur de signaler qu'il constate par perception directe qu'il n'est pas le seul à se retrouver à cet endroit à ce moment-là :

(22) Je ne suis pas le seul à me retrouver ici en pleine nuit, à ce que je vois. (frTenTen12)

Mais quelle est la nature catégorielle de l'unité que constitue à ce que je vois ? Est-ce une locution?

Martin définit une locution comme « un syntagme figé, situé au-delà du mot et en-deçà de la phrase figée » (1997 : 292). Des critères de trois types sont proposés par l'auteur pour identifier la locution prototypique : critères qui concernent les restrictions sélectionnelles, la (non-)compositionalité et la valeur intensionnelle (non référentielle) de ses parties.

La locution prototypique est caractérisée tout d'abord par des restrictions sélectionnelles des mots qui la composent. Dans la locution adverbiale à bâtons rompus, (23)a., il est impossible de substituer à bâtons d'autres noms, comme badine, baguette...(23)b. La même chose vaut pour les autres éléments de la locution (cf. (23)c. et d.) :

(23) a. Elles discutèrent encore deux heures à bâtons rompus et rentrèrent pour le déjeuner. (frTenTen12)

b. Elles discutèrent encore deux heures *à badines / *à baguettes rompues [...]

c. Elles discutèrent encore deux heures *à bâtons cassés [...]

d. Elles discutèrent encore deux heures *sans / *aux bâtons rompus [...]

Avec à ce que je vois, les restrictions sélectionnelles ne sont pas aussi strictes, comme nous l'avons vu sous $\S 1$. Seul ce que y est non substituable. Martin signale toutefois que les restrictions sélectionnelles ne constituent pas une condition nécessaire pour avoir une locution: "plus on se rapproche des marges locutionnelles, plus les degrés de liberté 
augmentent » (1997 : 293). D'après ce premier critère, à ce que je vois se trouve donc à la marge de la catégorie des locutions.

Le deuxième type de critère concerne la non-compositionalité de la séquence. Une locution prototypique signifie en général plus que le sens additionné de ses composantes. Le sens de la locution à bâtons rompus n'est pas compositionnel, le tout signifiant « de manière décousue, sans suite » (Larousse en ligne). Si on compare à ce que je vois à à bâtons rompus, la différence est grande : il est possible de comprendre le sens de à ce que je vois à partir des mots individuels, avec une petite réserve peut-être pour le $a$ initial, et là c'est comme « [d]ans un grand nombre de locutions prépositives, [où] il est difficile de justifier le statut de la préposition initiale : à l'exception de, à l'instar de [...] aux yeux de»" (Gross 2006 : 7). Selon ce second critère, il est difficile de dire que à ce que je vois est une locution prototypique.

Le troisième critère d'identification d'une locution prototypique concerne la valeur intensionnelle et non référentielle de ses parties (1997: 293). Dans à bâtons rompus, on évoque des bâtons, mais sans qu'il n'y ait aucun bâton, et encore moins un bâton rompu. Dans à ce que je vois, aussi bien je vois que ce que ont une valeur référentielle. Selon ce critère, l'expression sous étude ici ne serait pas non plus une locution prototypique.

On peut conclure que, d'après les critères proposés par Martin (1997), et en dépit de la catégorisation grammaticale du TLFi, à ce que je vois n'est pas une locution prototypique. L'expression a plus les caractéristiques d'une collocation, telle que définie par Lamiroy \& Klein (2005: 149, nos gras) :

Il y aurait la tendance universelle pour certains éléments de la langue à apparaître volontiers ensemble, au point où ces combinaisons peuvent finir par être institutionnalisées : c'est le cas des collocations, associations préférentielles mais non contraignantes.

dont ils donnent comme exemples : abuser de la gentillesse de qqn, n'avoir rien à dire de plus, ça va passer, ça tient à peu de choses, on n'aurait jamais pensé ça et telle que définie par Cowie (1981:224) :

A collocation is by definition a composite unit which [sic] permits the substitutability of items for at least one of its constituent elements (the sense of the other element, or elements, remaining constant).

Hausmann (1997 : 282) ajoute à cela que les collocations sont transparentes et que leur sens est compositionnel : les collocations sont «idiomatiques au niveau de l'encodage ». L'auteur illustre cela par la collocation rompre le silence. Un étranger ne peut pas prévoir que c'est bien le verbe rompre qui «met fin au silence », et non casser. Il nous semble que à ce que je vois présente, lui aussi, un certain encodage idiomatique : il est difficile en effet de prévoir notamment la préposition initiale.

Bref, les propriétés de à ce que je vois sont davantage celles d'une collocation (fréquence élevée des parties pour apparaître ensemble, sens compositionnel et idiomatique au niveau de l'encodage) que d'une locution prototypique. Nous y référerons donc par la suite au moyen du terme de collocation.

\section{3. À ce que je vois, marqueur d'évidentialité}

À ce que je vois est resté peu étudié en linguistique jusqu'à ce jour. Notre intérêt pour l'expression vient du fait qu'elle contient le verbe voir, verbe de perception, qui joue un rôle important à l'intérieur de l'étude du marquage évidentiel. Un marqueur évidentiel est un item - nécessairement grammatical, pour certains (Guentchéva 1996, Lazard 2001, 
Aikhenvald 2004), possiblement lexical pour d'autres (Squartini 2008) - qui signale comment le locuteur a pu se procurer l'information qu'il communique dans son énoncé. Un marqueur évidentiel signale donc la nature de la "source du savoir» (Dendale \& Tasmowski 1994) ou la «source de l'information » (Sapir 1921 : 114 -115, Bybee 1985 : 184, Chafe \& Nichols 1986 : partim). Les études qui proposent une classification des sources du savoir (p.ex. Willett 1988) retiennent en général trois grandes souscatégories évidentielles : la perception directe, l'emprunt à autrui et l'inférence (basée ou non sur des éléments perçus de la réalité). La perception revient donc deux fois dans les classifications des marqueurs d'évidentialité : dans la perception directe et dans l'inférence. Dans les trois sous-sections qui suivent ( $\$ 3.1$ à 3.4), nous nous demanderons à quelle(s) sous-catégorie(s) évidentielle(s) peut bien appartenir à ce que je vois : la perception directe, l'inférence ou l'emprunt.

\subsection{La perception directe}

La sous-catégorie évidentielle à laquelle à ce que je vois devrait appartenir prototypiquement - vu la présence justement de je vois - est celle de la perception directe. Or, dans notre corpus, la valeur de perception directe est loin d'être aussi courante qu'on ne l'aurait cru : 4,42\% seulement des occurrences. Quelques exemples :

(24)Les clefs en main je montais lentement les escaliers, je croisais la voisine qui habitait en face, elle était loup-garou, elle me gardait souvent quand j'étais enfant.

- Oh Lera, tu es de retour à ce que je vois ! Je souris.

- Bonjour madame Moonlane, vous allez bien? (frTenTen12)

(25)Le châtelain saisissant un flacon de whisky aux trois quarts plein, lui dit : «Vous avez une montre à secondes à ce que je vois. Quand je dirai : allez, faites démarrer l'aiguille, la bouteille je la retourne et la pose sur mon index, en équilibre. (Rheims, 1987, Frantext)

(26) Dis donc. Amadou! s'écria le sergent Mayclaire. Tu as maintenant un cheval, à ce que je vois! (Bâ, 1991, Frantext)

Pour des cas comme (27), on est en droit de se demander si on peut vraiment parler de perception directe. Est-ce que catégoriser quelqu'un comme un cavalier, relève de la perception directe ou est-on alors déjà dans l'inférence sur la base d'éléments perçus ?

(27) Même au bruit de la scie sur l'os, il poussa un cri : «On ne hurle pas, mon colonel! Maintenant on serre les quenottes, je suis sur le muscle. À ce que je vois, vous êtes cavalier, allons, ça ne fait pas si mal que ça. » (Rheims, 1987, Frantext)

Signalons encore des exemples intéressants comme (28)-(30), où la perception n'est pas de type visuel, mais tactile, auditif ou autre. Il s'agit là d'une extension assez commune de la signification de voir, qui va de la perception visuelle à la perception par d'autres sens, chose qui avait été observée aussi pour visiblement par Vanderheyden \& Dendale (2018: 18) :

(28) Oh, le vent se lève à ce que je vois, avait dit Drago Malefoy, accompagné de ses gorilles, à l'adresse de la jeune bulgare. Effectivement, le vent s'était levé davantage. (frTenTen12)

(29) Goomélie : Assistante Goomélie, vous êtes bien Toad? Toad : Bien sûr ! Qué vous pensez qué c'est qui ? Goomélie : Vous parlez d'un étrange dialecte à ce que je vois Toad: Ilci, peul-être qu'on pale pas mon langalge et je m'enl fou (frTenTen12)

(30) Alex pousse la porte et rentre dans la chambre, aperçois Alicia devant son bureau avec des écouteurs sur les oreilles Alicia chante Alex arrive par derrière et lui 
enlève ses écouteurs : T'as toujours une aussi belle voix à ce que je vois ou plutôt à ce que j'entends. (frTenTen12)

\subsection{L'inférence}

La fonction que à ce que je vois remplit le plus souvent en contexte (dans 94,26\% des occurrences de notre corpus) est d'indiquer que le locuteur (éventuellement représenté) a effectué une inférence à partir d'éléments qu'il a perçus (événements, comportements, propriétés). La collocation a alors une valeur évidentielle inférentielle. Le ce que de la collocation réfère aux indices sur lesquels le locuteur se base pour son inférence (qu'il faut le plus souvent reconstruire ou deviner, car ils sont rarement explicités). Voici un exemple où le cotexte (souligné) donne des informations qui peuvent très bien être interprétées comme les indices sur lesquels le locuteur a basé la conclusion inférée «vous donnez une soirée $»$ :

(31) Il était arrivé devant sa maison, il entra. La porte de la loge était ouverte, $\underline{\text { il vit sa }}$ concierge qui mangeait des marrons et buvait du vin blanc en compagnie de quelques voisines. - Bonsoir, madame, dit-il ; vous donnez une soirée à ce que je vois. (frTenTen 12)

Le caractère évidentiel inférentiel de à ce que je vois peut être justifié de diverses manières.

Premièrement, par le fait que le contenu des exemples avec à ce que je vois inférentiel décrit presque toujours des états de choses qui ne sont pas directement perceptibles : états de choses internes, états de choses passés ${ }^{7}$, évaluations, généralisations au-delà du perçu :

(32) Le style roman policier ou série noire te convient bien, à ce que je vois, tu y sembles très à l'aise et j'en suis très très amusée. (frTenTen12)

(33) Paco a beaucoup progressé en poussette, à ce que je vois. (frTenTen12)

(34) Pffffffff ces hommes, tous les mêmes à ce que je vois !!! Eux peuvent être crevés mais pas nous, c'est le comble! Sous prétexte que EUX travaillent! Nous on ne fait rien!!!! La vaisselle, la lessive, le nettoyage, le repassage, les courses, la tenue de la maison, les repas, s'occuper de bébé : manger, langer, bain, les calmer etc, s'occuper d'eux (ben oui) tous ça [sic] se fait tout seul!!!!! (frTenTen12)

Dans (32), il s'agit d'un état de choses interne à quelqu'un d'autre que le locuteur (une préférence qu'il a : «te convient bien »); il est inféré à partir de signes externes (non mentionnés dans l'extrait). Dans (33), le locuteur évalue le progrès que Paco a fait, sans probablement que le locuteur l'ait observé tout le temps. Il s'agit d'une évaluation, qui nécessite une comparaison de deux états de choses qu'il a perçus, donc une forme d'inférence. Dans (34), le locuteur généralise, par induction, à toute la classe d'hommes, se basant probablement sur un nombre limité d'observations.

Une deuxième indication du caractère inférentiel de à ce que je vois est dans la préposition initiale $\grave{a}$, qui peut être remplacée par avec, une préposition qui peut signaler la concomitance des indices et de la conclusion, voire " l'instrumentalité » des indices par rapport à la conclusion. Le groupe prépositionnel avec ce que je vois, signifie «avec les choses/indices que je vois, je peux dire/conclure que ", qui invite à une lecture inférentielle :

(35) Goldmos, la raison pour laquelle je voulais que ce loup participe a la mission c'est simplement pour qu'il puisse nous montrer ce qu'il sais faire. Pour qu'il puisse ainsi monter dans notre estime. Mais avec ce que je vois, il ne veux rien de notre estime. (frTenTen 12 ; orthographe originelle maintenue) 
(36) Je suis profondément convaincu qu'on se dirige vers la civilisation de l'amour. Avec ce que je vois, je réalise que c'est en marche. (frTenTen12)

Pour le TLFi (s.v. à (sous F.1.c.)), à introduit un « complément d'indice ou de cause », par exemple après un verbe de perception ou d'entendement : reconnaître quelqu'un au pas ("par le pas, à cause de son pas »), je vois ça à des tas de petits trucs (« sur la base de petits trucs »). Le à de à ce que je vois pourrait bien avoir cette fonction-là ("sur la base de ce qu'on voit»). $\grave{A}$ ce que je vois réfère ainsi à l'évidentialité inférentielle par l'intermédiaire d'un renvoi à l'« input » de l'opération inférentielle, la base de l'inférence (ses indices).

Troisième indication: la collocation est sémantiquement fort proche de l'adverbe visiblement, qui a été décrit, dans son emploi exophrastique, comme un marqueur évidentiel inférentiel par Vanderheyden \& Dendale (2018) et Dendale, Vanderheyden \& Izquierdo Alegría (2020). Dans la majorité des occurrences de à ce que je vois dans son emploi comme collocation citées dans cette étude, il est possible de substituer visiblement à à ce que je vois. Les principales exceptions, pour nous, sont: (a) les cas où à ce que je vois exprime non l'inférence, mais la perception directe (ex. (24)-(26)) et $(38)^{8}$; (b) certains cas d'extension de la vue à d'autres sens (ex. (29) et (30), encore que visiblement admette en principe cette extension); (c) les cas où à ce que je vois est suivi d'un [CCirc], pour des raisons sans doute syntaxiques (les exemples (14) et (40) et celui de la note 4$)^{9}$. Les deux différences principales entre les expressions nous semblent être: (i) que à ce que je vois apparaît nettement plus souvent en tête de phrase que visiblement et (ii) qu'avec visiblement on exprime plutôt la bonne visibilité (cf. Dendale, Vanderheyden \& Izquierdo Alegría (2020)) et par conséquent une prise en charge de la vérité du contenu assez forte, alors qu'avec à ce que je vois, c'est plutôt la visibilité limitée (voir §4) et la diminution de la fiabilité de l'information (voir $\S 5$ ).

Une quatrième indication du caractère inférentiel de à ce que je vois vient de sa proximité avec les verbes paraître et sembler, dont le caractère évidentiel inférentiel a également été reconnu pour diverses langues.

\subsection{Entre perception directe et inférence. Le cas de la photo}

Un cas de figure intéressant est formé par les exemples où à ce que je vois se rapporte à ce que le locuteur voit ou a pu voir sur une photo. La question est de savoir s'il y est question alors d'une perception directe de l'état de choses décrit dans l'énoncé, ou d'une inférence de cet état de choses à partir de ce que le locuteur voit sur la photo. Dans le dernier cas, la photo serait ou contiendrait l'indice ou les indices à partir desquels le locuteur tire une conclusion par inférence. Considérons quelques exemples :

(37) Ça me fait plaisir de voir ces photos, elle est en forme à ce que je vois, et elle a pas perdu son petit ventre. (frTenTen 12)

(38) Ces photos sont vraiment belles. Le restaurant des oiseaux est plein à ce que je vois. Toute cette période de grand froid n'a pas dû être facile pour les animaux. (frTenTen12)

(39) Très chouettes tes nouvelles photos ! Tu t'es bien amusée à ce que je vois. (frTenTen 12)

(40) A ce que je vois à travers les photos les paysages sont magnifiques, ouvre grand tes yeux et profites de chaque instant. (frTenTen12)

On notera sur ce point une différence entre (37)-(38) d'un côté et (39)-(40) de l'autre. Dans les deux derniers exemples, l'insertion d'un devoir épistémique, marqueur évidentiel d'inférence, est possible et naturelle : tu dois t'être amusée/tu as dî t'amuser; les paysages doivent être magnifiques. Cette insertion reviendrait à faire de ce que le locuteur voit sur la 
photo des indices qui lui permettent de conclure à ce qui est décrit dans ces énoncés. $\grave{A}$ ce que je vois y signale une inférence plutôt que la perception directe d'états de choses. Cette interprétation est confirmée par le fait que s'amuser dans (39) désigne une activité que l'on peut difficilement constater sur une image statique et que ce verbe comprend en plus un élément d'évaluation, évaluation à laquelle l'on ne peut parvenir que sur la base d'indices. Dans (40), c'est la locution prépositionnelle à travers qui contribue à l'interprétation comme inférence. On y parle non pas des paysages sur la photo, mais de paysages réels d'ailleurs, dont on évalue la beauté à travers ce qui se trouve sur la photo : inférence donc plutôt que perception directe. Dans (37)-(38), une insertion de devoir épistémique paraît moins facile et moins naturelle ; les états de choses décrits sont des états de choses externes. Les photos sont des images " fidèles ${ }^{10}$ » de la réalité : regarder la photo équivaut à regarder la réalité et on peut donc parler dans ces cas de perception directe. $\grave{A}$ ce que je vois doit y être considéré comme un marqueur évidentiel de perception directe.

\subsection{Et l'emprunt?}

Il semble plutôt évident, vu la signification de voir, de ne pas catégoriser à ce que je vois comme un marqueur évidentiel d'emprunt ${ }^{11}$. Or, il y a deux constats dont il faut tenir compte.

Le premier, que l'on peut écarter assez facilement, concerne l'existence des variantes de la collocation construite sur le schéma (1), notamment celles où à la place de je vois, [PronPers] [Verbe], on a des verbes d'emprunt, $j$ 'ai appris, $j$ 'ai ouï dire, $j$ 'ai entendu ou on dit (11), etc., comme dans les exemples :

(41)Le toit de l'Arche de la Défense est inaccessible depuis un mois du fait d'une panne des ascenseurs panoramiques, ce qui fait que les entreprises qui y logent sont en arrêt technique. A ce que j'ai appris de source informée (mais non confirmée par la presse), il existe d'autres ascenseurs (pour couvrir les deux «flancs» de l'arche), mais les organisations dont dépendent ces ascenseurs refusent de partager l'accès. (frTenTen12)

(42) Allons ! il faudra que j'aille à Valençay. Il y a là de bons menuisiers, à ce que j'ai ouï dire. (frTenTen 12)

Le second, un peu plus « gênant » théoriquement parlant, est le cas de l'exemple (43) :

(43) [Un homme interroge Ueda Tatsuya sur la base d'un formulaire que celui-ci vient de remplir :]

Alors vous êtes Ueda Tatsuya, 28 ans?

Oui, c'est ça.

Hum... Né à Kanagawa à ce que je vois.

En effet. (frTenTen12)

Sur le formulaire, Tatsuya avait noté qu'il est né à Kanagawa. Son interlocuteur, qui vient de prendre connaissance du contenu du formulaire dit qu'il voit que l'interrogé est né à Kanagawa. À ce que je vois doit s'interpréter dans ce contexte comme «à ce que je lis ", variante écrite de j'ai appris, on m'a dit,... Lire, apprendre, dire sont tous des verbes qui réfèrent à une opération d'emprunt plutôt qu'à une opération de perception ou d'inférence. Ce que l'interlocuteur voit ce n'est pas la réalité extérieure, ni une image ou photo, mais un bout de texte. Il ferait donc un certain sens de considérer à ce que je vois, en tout cas dans des cas très spécifiques comme (43), comme un marqueur évidentiel d'emprunt : selon ce que tu as écrit / à tes dires, tu es né à Kanagawa. La « source de l'information » sur le lieu de naissance de Tatsuya est un dire (un «écrire ») de Tatsuya emprunté par le locuteur (représenté). 
Il y a toutefois encore une autre interprétation possible de cet exemple et de l'opération de l'acquisition d'information qu'il y a derrière à ce que je vois. L'interrogateur n'a évidemment pas assisté à la naissance de Tatsuya et n'a donc pas eu de perception directe de la naissance à Kanagawa. La déclaration écrite de Tatsuya peut aussi être prise pour un indice qui permet de conclure qu'il est né à Kanagawa : " de ce que vous avez noté sur votre formulaire, je conclus que vous êtes né à Kanagawa». Dans ce cas, à ce que je vois pourrait également être pris comme un simple cas d'inférence.

\section{4. À ce que je vois versus à ce que je peux voir}

Dans certains emplois de à ce que je vois, on a du mal à se défaire de l'impression que la collocation invite à lui attribuer un élément de sens supplémentaire, qui s'ajoute à celui de l'expression de l'évidentialité (§3). Cet élément est que la perception du locuteur a pu être limitée ou entravée. Ce trait est paraphrasable comme : «(du moins) pour autant que je puisse / que j'aie pu voir ». On le trouve sous une variante fort explicite dans :

(44) Mais le visage du médecin restait scellé.

- Pas d'autres taches sur le corps?

- Non, du moins à ce que j’ai pu voir. À l'hôtel, il n'avait pas osé examiner son corps, de crainte d'y découvrir de nouvelles taches (Montherlant 1939, Frantext)

Nous allons d'abord essayer d'expliquer d'où peut venir ce trait et ensuite $(\S 5)$ montrer quel pourrait en être son effet sur le sens global des énoncés contenant à ce que je vois.

Il nous apparaît que cette impression de perception limitée ou entravée pourrait être liée à la présence (effective ou virtuelle), à l'intérieur de la collocation, du semi-auxiliaire modal pouvoir, au sens de "capacité ». Parler de ce qu'on peut voir, c'est-à-dire de ce qu'on est en mesure de voir c'est évoquer, pragmatiquement, qu'il y a des choses que l'on n'a (éventuellement) pas pu voir. En d'autres termes, c'est évoquer les limites de sa propre perception, donnant ainsi à la collocation le sens de "pour autant que je puisse voir ». La perception limitée peut aussi avoir à faire avec le sens de d'après (qu'évoque le à initial), qui suggère, globalement, l'existence d'une autre perspective ou d'une autre base pour une conclusion :

(45) Mais sur la photo on peut aussi apercevoir trois objets suspects (notés A, B et C cidessous) dans les anneaux de la planète. A priori ils seraient aussi sur Mars d'après cette photo (frTenTen 12)

Il a été montré (cf. Le Querler 1989) que dans des contextes bien définis il y a une quasi-équivalence sémantique entre je vois $X$ et Je peux voir $X$, par exemple entre (46)a. et b. :

(46) a. D'ici on voit la mer.

b. D'ici on peut voir la mer. (Le Querler 1989 : 71)

Il y a à cela deux conditions, décrites par Le Querler :

Première condition. Le sujet désigne un individu quelconque : on, tout le monde, n'importe qui ou un SN défini avec article générique. Cette condition est remplie dans (46), de même que dans (47) :

(47)a. D'ici le promeneur / le touriste / les gens / les randonneurs voit / voient la mer b. D'ici le promeneur / le touriste / les gens / les randonneurs peut voir / peuvent voir la mer. (Le Querler 1989 : 71)

Les énoncés (46) et (47) signifient « quand on est ici, on a vue sur la mer, aucun obstacle n'empêche qu'on la voie » (Le Querler 1989: 72). Ces énoncés assertent que «quelque 
chose est "perceptible", par n'importe qui qui se trouve 'là' » (1989: 72). Ils assertent donc une propriété de l'endroit (ici) ou une propriété de l'objet de la perception (la mer)», un peu comme le fait l'adjectif visible. Ils n'assertent pas en revanche une propriété de l'agent qui perçoit, d'où que le sujet de voir peut désigner un individu quelconque (p.ex. on) et qu'il est possible de le remplacer par un SN défini générique. Cette première condition ne semble toutefois pas être remplie dans le cas de à ce que je vois. Difficile de dire que je est un sujet quelconque. Mais comme une sorte d'exception à cette première condition, Le Querler note que le sujet du verbe de perception peut être le pronom personnel je à condition que l'énoncé puisse être interprété comme l'assertion d'une propriété occasionnelle du sujet du verbe voir (et non une propriété permanente de son sujet). Cette condition est remplie dans les énoncés sous (48), (qui selon Le Querler (1989 : 74) assertent une propriété occasionnelle du sujet), mais pas dans ceux sous (49) (qui assertent une propriété permanente) :

(48)a. Machin, je le vois quand je veux.

b. Machin, je peux le voir quand je veux. (Le Querler 1989 : 74)

(49)a. Je vois mieux avec mon nez qu'avec mes yeux.

b. 'Je peux mieux voir avec mon nez qu'avec mes yeux. (Le Querler 1989 : 73)

Le propre des propriétés occasionnelles est qu'elles ont toujours un ancrage spatio-temporel et que l'énoncé avec pouvoir est possible. C'est ce qu'on observe aussi avec à ce que je vois :

(50) Le docteur Brosseau fit son apparition [...]

- Bonjour, mes enfants. Ha ha! Un petit abcès, à ce que je vois. C'est moins que rien : je t'arrange ça tout de suite, ma fifille... Ouvre la bouche bien grand... (Beauchemin, 1981, Frantext)

Exprimant une propriété occasionnelle du sujet, à ce que je peux voir est possible et équivalent de à ce que je vois. Nous concluons que la première condition est remplie.

Deuxième condition. La perception exprimée par le verbe est involontaire ${ }^{12}$. Cela veut dire, premièrement, qu'on aura donc voir plutôt que regarder, entendre plutôt que écouter et, deuxièmement, que le verbe accepte sans problème des adverbes comme facilement, sans peine, difficilement, mais plus difficilement délibérément, volontairement. Cette condition est remplie dans l'exemple suivant, où la personne désignée par $t u$ n'a pas le choix de « voir ou ne pas voir que le locuteur est présent ». La perception est involontaire : elle ne nécessite pas «d'intervention de la volonté du sujet » (1989:75) :

(51) a. Comme tu le vois, je suis à l'heure.

b. Comme tu peux le voir, je suis à l'heure. (Le Querler 1989 : 75)

Cette condition explique aussi pourquoi on trouve des à ce que je vois et à ce que j'entends, mais pas des à ce que je regarde ${ }^{13}$ et à ce que j'écoute. Voir (mais aussi entendre et sentir) sont des verbes «expérientiels»; regarder (et écouter) des verbes «agentifs", qui «décrivent non une expérience perceptive mais une activité du sujet de perception portant intentionnellement sa vue, son regard... vers l'objet-cible» (Benzakour 1993 : 46).

Nous concluons que les deux conditions de quasi-équivalence de voir avec pouvoir voir sont remplies dans le cas de l'expression à ce que je vois : à ce que je vois équivaut donc grosso modo, à à ce que je peux voir. On constate une équivalence similaire dans l'adverbe visiblement, qui, par son suffixe de possibilité -ible signifie «d'après / de] ce [qu'on peut / que je peux] voir », mais qui comme adverbe évidentiel signifie « d'après ce que je vois ». 
En utilisant pouvoir, au sens de capacité, auprès de voir, on signale donc les limites de la perception, les limites de ses propres capacités.

Dans la section qui suit, nous allons voir l'effet possible de l'interprétation de à ce que je vois comme «à ce que je peux voir ».

\section{De la perception limitée à la conclusion défectible}

Dans les contextes où l'on peut interpréter à ce que je vois, marqueur évidentiel de perception directe, comme "d'après ce que je peux voir », ou l'on utilise une variante de la collocation qui contient le verbe pouvoir, on peut avoir l'impression que la collocation suggère que la perception a pu être entravée. Cela peut être dû à l'organe de perception (problème d'yeux), au canal (perception à distance, perception dans obscurité ou le brouillard, perception avec obstacles, champ de vision restreint), à l'acte de perception (trop rapide et superficiel) ou à la situation observée (p.ex. situation qui évolue). Prenons (52) :

(52) Vous êtes seul, à ce que je vois. (frTenTen12)

À ce que je vois y a deux interprétations possibles : « je vois » et «pour autant que je puisse voir / à ce que je peux voir ». Cette deuxième interprétation revient à suggérer que la perception pourrait être trompeuse (qu'il est possible que d'autres personnes viennent s'ajouter plus tard). La conséquence de tout cela est que ce qui paraît être le cas pourrait très bien s'avérer (après suppression des entraves à la perception) ne pas être le cas. La présence de la collocation peut donc faire naître chez l'interlocuteur un doute quant à la fiabilité de l'affirmation communiquée.

La limitation de la perception a aussi des conséquences lorsque à ce que je vois est utilisé comme marqueur évidentiel inférentiel. Puisque dans ce cas, la perception concerne les indices, elle aura une conséquence pour la fiabilité des indices qui sont à la base de la conclusion que le locuteur tire. La conséquence en est que l'inférence est présentée comme « défectible » (defeasible), selon la définition suivante de cette notion :

We reason defeasibly when we reach conclusions that we might be forced to retract when faced with additional information. (Nute, $1988: 251$ )

S'il s'avère que les indices sur lesquels le locuteur s'est basé sont le résultat d'une perception erronée, la conclusion en subit les conséquences et devra probablement être corrigée.

L'interprétation comme perception limitée (avec toutes les conséquences que cela peut avoir, y compris le raisonnement défectible) n'est qu'une interprétation possible. Elle est relativement facile à détecter lorsque le marquage est explicite: par l'ajout du verbe pouvoir (53), ou par l'ajout d'un marqueur de restriction (du moins, enfin) (54)-(55), ou encore par la substitution à à d'une autre préposition initiale (après ou selon) (56) :

(53) Enfin, à ce que je peux voir, il est mort. Blessure par balle. (frTenTen12)

(54) Je suis bien content que ses prières aient été exaucés [sic], mais, en même temps, je pensais que vous étiez... plus grand et plus imposant. Mais ce n'est pas le cas, du moins, à ce que je vois. (frTenTen 12)

(55) Puck est un très bon père pour Beth, enfin à ce que je vois. Elle a l'air heureuse avec eux donc c'est ça qui compte. (frTenTen 12)

(56) D’après ce que je vois : tu vis ici, tu es serveur et célibataire ! (frTenTen12)

Lorsque ce marquage explicite manque, elle est parfois plus difficile à détecter, par exemple dans (24) à (26). Beaucoup dépend de la situation décrite et de l'éventuelle 
difficulté d'observation qu'elle amène. Dans (44) ou (50) par exemple, elle est plus facilement concevable.

\section{Conclusion}

Dans cette étude, nous nous sommes d'abord interrogés sur la nature grammaticale de à ce que je vois : locution ou non? Étant donné l'existence des nombreuses variantes de la suite de mots $\grave{a}+c e+q u e+j e+$ vois que l'on peut lister à partir du schéma de construction « [Prép] ce que [PronPers] [Verbe] ([CCirc]) » et vu les trois critères d'identification d'une locution prototypique de Martin (1997), nous avons conclu que ce groupe de mots n'est pas une locution, en tout cas pas prototypique, mais plutôt une collocation. C'est donc par ce termelà que nous l'avons désigné.

Le but principal de cette étude a consisté à montrer que à ce que je vois, lorsqu'il a le statut de collocation à fonction d'adverbial de phrase, peut être considéré comme un marqueur évidentiel. Nous avons montré qu'il appartient le plus clairement et le plus souvent à la catégorie des marqueurs d'évidentialité inférentielle sur la base d'indices perçus (visuels ou, par extension, auditifs ou tactiles), les indices étant ce à quoi réfère ce que dans la collocation. Plus exceptionnellement, la collocation appartient à la catégorie des marqueurs évidentiels de perception directe.

Nous avons ensuite examiné un élément qui accompagne souvent le sens évidentiel de la collocation, la limitation possible de la perception. Cet élément de sens est lié à la présence implicite ou explicite du verbe pouvoir dans la collocation et à l'interprétation de la préposition initiale à comme "d'après ». Il est responsable du caractère défectible de la conclusion lorsque la collocation fonctionne comme marqueur évidentiel d'inférence. La limitation de la perception influence aussi la fiabilité de l'information communiquée.

\section{Références bibliographiques}

Aikhenvald, A. (2004). Evidentiality. Oxford : Oxford University Press.

Anscombre, J.-Cl. (2013). Entité lexicale : apparemment, in Anscombre, J.-Cl., Donaire, M. L. \& Haillet, P. P. (éds), Opérateurs discursifs du français. Éléments de description sémantique et pragmatique, Bern, Lang, p. 49-62.

Benzakour, F. (1993). Les compléments de comptes rendus de perception : Quelques cas en français. L'information grammaticale, 56, p. 46-48.

Bybee, J. (1985). Morphology: A study of the relation between meaning and form. Amsterdam: John Benjamins.

Chafe, W. \& Nichols, J. (éds), (1986). Evidentiality: The Linguistic Coding of Epistemology. Norwood: Ablex Publishing Corpor.

Cowie, A. P. (1981). The treatment of Collocations and Idioms in Learner's Dictionaries. Applied linguistics, 2.3, p. 223-235.

Delattre, P. (1939). Accent de mot et accent de groupe. The French Review, 13.2, p. 141-146.

Dendale, P. \& Tasmowski, L. (1994). Présentation : l'évidentialité ou le marquage des sources du savoir. Langue française, 102, p. 3-7.

Dendale, P., Vanderheyden, A. \& Izquierdo Alegría, D. (2020). Visiblement en français, visiblemente en espagnol : des marqueurs évidentiels de perception directe ou d'inférence ? in Loureda, Ó., Rudka, M. \& Parodi, G. (éds.), Marcadores del discurso y lingüistica contrastiva en las lenguas románicas, Iberoamericana/Vervuert, p. 63-96.

Gross, G. (2006). Sur le statut des locutions prépositives. Modèles linguistiques, 53, p. 35-50.

Guimier, C. (1996). Les adverbes français : le cas des adverbes en -ment. Paris : Ophrys.

Guentchéva, Z. (1996). L'énonciation médiatisée. Louvain : Peeters.

Hausmann, F. J. (1997). Tout est idiomatique dans les langues. in Martins-Baltar, M. (éds.), La locution: entre langue et usages, Fontenay-aux-Roses : ENS Éditions, p. 277-290. 
Lamiroy, B. \& Klein, J.R. (2005). Le problème central du figement est le semi-figement. Linx, 53, p. $135-154$.

Lazard, G. (2001). On the grammaticalization of evidentiality. Journal of Pragmatics, 33, p. 359-367.

Le Querler, N. (1989), Quand voir, c'est pouvoir voir. Langue française, 84, p. 70-82.

Martin, R. (1997). Sur les facteurs du figement lexical. in Martins-Baltar, M. (éds.), La locution : entre langue et usages, Fontenay-aux-Roses : ENS Éditions, p. 291-305.

Martins-Baltar, M. (1997). Repères dans les recherches actuelles sur la locution. in Martins-Baltar, M. (éds.), La locution: entre langue et usages, Fontenay-aux-Roses : ENS Éditions, p. 19-53.

Nute, D. (1988). Defeasible reasoning: a philosophical analysis in prolog. Aspects of artificial intelligence. Dordrecht : Springer, p. 251-288.

Sapir, E. (1921). Language: An introduction to the study of speech. New York : Hardcourt, Brace.

Squartini, M. (2008). Lexical vs. grammatical evidentiality in French and Italian. Linguistics, 46.5, 917-947.

Svensson, M. H. (2004). Critères de figement : L'identification des expressions figées en français contemporain. Thèse de doctorat. Umeå, Umeå : University.

TLFi: Trésor de la langue française informatisé, http://www.atilf.fr/tlfi, ATILF - CNRS \& Université de Lorraine.

Vanderheyden, A., \& Dendale P. (2018). Visiblement: l'évolution diachronique d'un marqueur évidentiel. Zeitschrift für romanische Philologie, 134.4, p. 1008-1035.

Willett, Th. (1988). A cross-linguistic survey of the grammaticization of evidentiality. Studies in Language, 12.1, 51-97.

${ }^{1}$ Dieguez, Sebastian, 2013, "Dans la tête de Sherlock Holmes", Cerveau \& Psycho, 59, https://www.cerveauetpsycho.fr/sd/cognition/dans-la-tete-de-sherlock-holmes-7527.php.

${ }^{2}$ Une recherche dans frTenTen 12 a montré toutefois que les variantes en $t u$, nous et on sont peu fréquentes. La variante à ce que tu vois représente $2 \%$ des occurrences de «à ce que [Pronom Personnel] vois »; pour à ce que nous voyons et à ce qu'on voit, c'est $4 \%$.

${ }^{3}$ Pour les deux derniers types de verbes, il y a aussi le pronom sujet qui change.

${ }^{4}$ Ou encore un complément déterminatif du pronom ce comme dans :

Dis-moi: où sommes-nous, que fais-je ici, pourquoi tout est si clair, pourquoi n'y a t'il [sic] pas de sol, pourquoi ne puis-je te voir et d'abord où es-tu? - Allons, du calme, nous avons encore un peu de temps, à ce que je vois de ton sablier... (FrTenTen12)

5 Cette proposition peut être elliptique: Une enfance terrible, à ce que je vois. (frTenTen12). Dans notre corpus de travail, 12,83\% des occurrences de à ce que je vois sont incidentes à des propositions elliptiques.

${ }^{6}$ Dans notre corpus de 500 occurrences, à ce que je vois postposé est de loin le plus fréquent : $63,4 \%$ des occurrences versus $24,4 \%$ pour les occurrences de à ce que je vois antéposé et $2,4 \%$ des occurrences de à ce que je vois en position d'incise médiane.

${ }^{7}$ Contrairement à ce qu'on observe avec d'autres marqueurs évidentiels inférentiels (visiblement, certainement, devoir...), à ce que je vois ne se combine pas (d'après les recherches que nous avons faites) avec des états de choses futurs.

${ }^{8}$ Dans (37), il nous semble que visiblement forcerait une lecture inférentielle. Cela paraît plus difficile pour à ce que je vois (pour des raisons qu'il reste à élucider).

${ }^{9}$ Les occurrences de à ce que je vois en combinaison avec enfin et du moins mériteraient une analyse plus poussée sur ce point. Si on y remplace la collocation par visiblement, on crée apparemment une opposition entre l'être et le paraître, comparable à celle qu'on la trouve avec l'adverbe apparemment : enfin visiblement et du moins visiblement en position finale de phrase, ajoutés comme une sorte de correction de ce qui précède, paraissent prendre le sens de «en apparence seulement » qu'Anscombre (2013 : 53) avait relevé pour cet adverbe (son emploi apparemment $)_{2}$ ). La conséquence est que visiblement s'éloigne, dans ce cas, de son emploi comme marqueur évidentiel. C'est quelque chose que Vanderheyden \& Dendale (2018) et Dendale, Vanderheyden \& Izquierdo Alegría (2020) n'ont pas relevé dans leurs études sur visiblement. 
${ }^{10}$ Photoshop mis à part...

${ }^{11}$ À l'exception d'une des sous-significations que mentionne le TLFi, à savoir « percevoir à travers/ par les yeux de quelqu'un » comme dans :

Je laissai retomber le rideau. Qu'avais-je espéré? Voir un instant avec les yeux de Paule ce décor familier? saisir sur ce décor la couleur de ses jours? (de Beauvoir, 1954, cité d'après le TLFi).

${ }^{12}$ Cf. la définition de l'acception I.A.1.a) $\alpha$ de voir par le TLFi (s.v. voir) : «Enregistrer l'image de ce qui se trouve dans le champ visuel, d'une manière passive, sans intention préalable $»$ (nos gras).

${ }_{13}$ À l'exception des deux exemples rares avec à ce que j'ai pu regarder, que Danielle Coltier nous a aimablement fournis :

Car pour le coup, à ce que j'ai pu regarder, on est plutôt sur un boitier seul ou limite. (Flickr)

Eu égard aux réponses données et à ce que j'ai pu regarder rapidement, il sera au forfait et pour la TVA on verra. (Compta-online) 